\title{
Reduction of the turbulent blob transport in the scrape-off layer by a resonant magnetic perturbation in TEXTOR
}

\author{
Y. Xu ${ }^{1}$, R. R. Weynants ${ }^{1}$, M. Van Schoor ${ }^{1}$, M. Vergote ${ }^{1}$, S. Jachmich ${ }^{1}$, \\ M. W. Jakubowski ${ }^{2,3}$, M. Mitri ${ }^{2}$, O. Schmitz ${ }^{2}$, B. Unterberg ${ }^{2}$, P. Beyer ${ }^{4}$, \\ D. Reiser ${ }^{2}$, K. H. Finken ${ }^{2}$, M. Lehnen ${ }^{2}$, and the TEXTOR Team \\ 1 Laboratoire de Physique des Plasmas - Laboratorium voor Plasmafysica, \\ Association 'Euratom-Belgian state', \\ Ecole Royale Militaire - Koninklijke Militaire School, \\ Trilateral Euregio Cluster, B-1000 Brussels, Belgium \\ ${ }^{2}$ Institute für Energieforschung-Plasmaphysik, \\ Forschungszentrum Jülich, Association EURATOM/FZJ, \\ Trilateral Euregio Cluster, D-52425 Jülich, Germany \\ ${ }^{3}$ Max-Planck-Institut für Plasmaphysik, \\ IPP-EURATOM Association, Teilinstitut Greifswald, \\ Wendelsteinstr. 1, 17491Greifswald, Germany and \\ 4 Equipe Dynamique des Systemes Complexes, PIIM, \\ CNRS-Universite de Provence, Centre de St. Jerome, \\ Case 321, 13397 Marseille Cedex 20, France
}

(Dated: January 9, 2009)

\begin{abstract}
During the static 6/2 Dynamic Ergodic Divertor experiments in TEXTOR, a significant influence of the edge resonant magnetic perturbation (RMP) on the turbulent blob transport in the scrape-off layer (SOL) has been observed. In ohmic discharges without the RMP, the blobs extend 4-5 cm deep into the SOL with a radially-outward moving speed of about $1 \mathrm{~km} / \mathrm{s}$, and hence, are constituting a strong outflow of mass. With the application of the RMP, the blob amplitudes and their radially moving velocity are both reduced, resulting in a significant reduction of the blob transport in the SOL. The reduction effect of the RMP on blobs is found to be robust to changes in the operational regime and to phasing variations of the RMP as well. The blob dynamics appears to be consistent with the paradigm of the radial motions of the blob structures driven by the interchange instability.
\end{abstract}




\section{INTRODUCTION}

Experimental data from tokamaks (and other fusion devices) show that the radial transport in the scrape-off-layer (SOL) has a mostly convective and intermittent character, usually referred to as blob transport [1-7]. A blob is a coherent structure extending in a filamentary way along the $\mathbf{B}$ field line, characterized by a density much higher than the ambient density and leaving the plasma at high radial velocity. Such bursty events can unfavorably increase the recycling at the main chamber walls, reduce the divertor effciency and may thus lead to a high level erosion of the first wall in fusion devices like ITER (International Thermonuclear Experimental Reactor) [8]. In recent years, experiments in several machines have shown the role of an ergodized magnetic boundary induced by an edge resonant magnetic perturbation (RMP) in mitigating edge-localized modes (ELMs) $[9,10]$ and also in reducing the plasma-wall interaction [11, 12]. During the static Dynamic Ergodic Divertor (DED) operation at TEXTOR, significant changes in turbulence properties and turbulence-driven transport have been observed in the edge plasma region, especially in the ergodic zone [13, 14]. In the ELM control experiments in DIII-D, the impact of the RMP on edge turbulence has also been reported $[15,16]$.

In this paper, we present the first experimental results on the effect of a RMP on the blob transport in the SOL of TEXTOR and it is shown that such transport is strongly reduced. The possible physical mechanisms for this action are also discussed.

\section{EXPERIMENTAL CONDITIONS AND SET-UP}

The experiments were mainly performed in ohmic deuterium discharges in TEXTOR having $\mathrm{R} / a \cong 1.73 / 0.46[\mathrm{~m} / \mathrm{m}], B_{T}=1.9 \mathrm{~T}, I_{p}=270 \mathrm{kA}$ and line-averaged densities in a range of $\bar{n}_{e}=(1.6-3.5) \times 10^{19} \mathrm{~m}^{-3}$. The edge magnetic perturbation was created by $16 \mathrm{DED}$ coils, helically wound around the inner side of the torus with a pitch parallel to field lines on the magnetic flux surface with a safety factor $q \cong 3$ [12]. For the present experiment, the base poloidal/toroidal modes were operated as $m / n=6 / 2$ with a $d c$ DED current, $I_{D E D}$, applied in the stationary phase of the ohmic discharge.

Typical discharge waveforms are plotted in Fig. 1. From Figs. 1(a) and (b), we can see that the $I_{D E D}$, ramping from 0 to $6 \mathrm{kA}$ and then held constant for $0.7 \mathrm{~s}$, is applied in the 


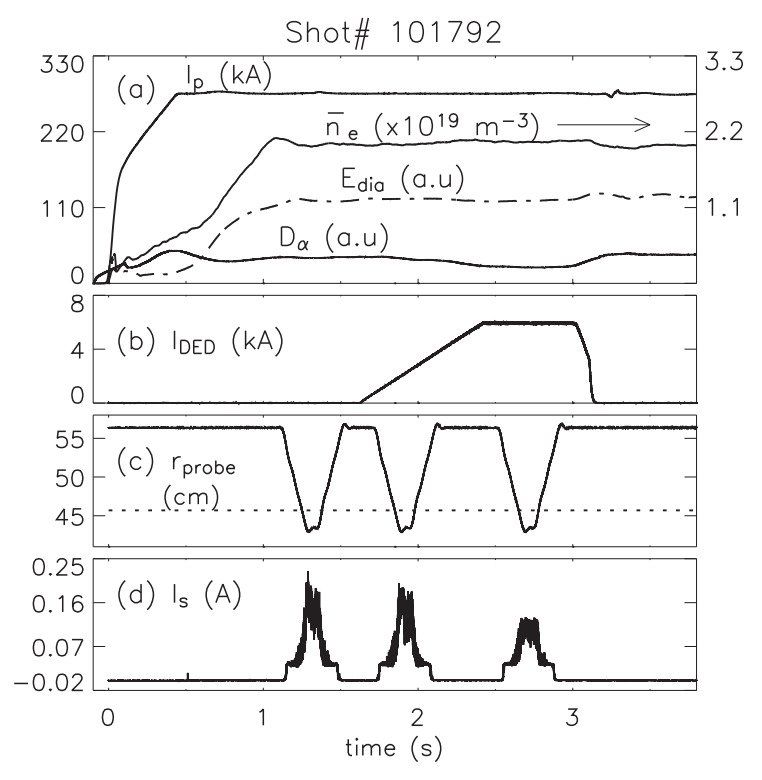

FIG. 1: Discharge waveforms in a static 6/2 DED experiment on TEXTOR. (a) Plasma current $I_{p}$, line-averaged density $\bar{n}_{e}$, diamagnetic loop signal $E_{\text {dia }}$ and $D_{\alpha}$ emission on limiter; (b) DED current; (c) radial position of fast reciprocating probes (dotted line denotes limiter location); (d) ion saturation current.

stationary discharge stage. The DED-induced edge RMP does not degrade the quality of the global confinement as $\bar{n}_{e}$ and the diamagnetic energy $E_{\text {dia }}$ signals both stay constant and the edge $D_{\alpha}$ signal reduces during the DED phase. A fast reciprocating Langmuir probe array [14], installed at the outer midplane (poloidal angle $=0$ ), was used to measure the blobs and the underlying plasma turbulence. Two pins of the array were DC-biased to record the ion saturation current, $I_{s}$, while two others were poloidally spaced to detect the poloidal electric feld $E_{\theta}$ from their floating potential difference. For the study of the RMP impact on the blob transport in the SOL, we plunged the fast probes three times during one shot for comparison: the first one before the DED, the second in ramp phase and the third in the plateau of $I_{D E D}$ (see Fig. 1(c)). For each plunge, the probe position reaches the plasma edge (the dotted line denotes limiter locus). The corresponding $I_{s}$ signal is plotted in (d).

During the plateau of $I_{D E D}(=6 k A)$, the DED current induces a perturbation field $B_{r} / B_{T} \approx 3 \times 10^{-4}$ at the resonant surface $(q=3)$ and thus causes stochastization of the magnetic field lines in the plasma boundary $[12,18]$. Figure 2 shows the corresponding Poincaré plot of the edge magnetic field lines, which is calculated based on an equilibrium field with the perturbing one superimposed in the vacuum approximation[18, 19]. Also shown is the 


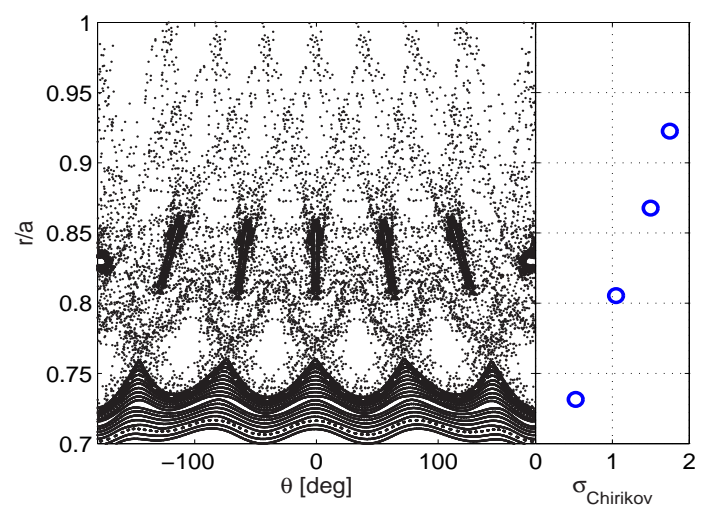

FIG. 2: Left: Poincaré plot of the edge magnetic field topology in the $(\mathrm{r} / a, \theta)$ plane for the plasma parameters of Fig. 1. Right: The radial profile of the Chirikov parameters.

radial profile of the Chirikov parameter, $\sigma_{\text {chirikov }}$, defined as the ratio of the averaged island width to the radial distance of adjacent island chains. The perturbation extends rather deep into the plasma, creating a "LCFS" around $\rho \equiv \mathrm{r} / a \approx 0.72$, surrounded by an ergodic zone $(\rho \approx 0.72-0.95)$, with long field-line connection lengths and extra transport related to field line diffusion, and a laminar zone $(\rho \approx 0.95-1.0)$, with short connection lengths and dominant parallel transport, respectively [18].

Note that in this investigation, we compare the experimental results with and without the DED at the same radial positions and a unique toroidal one. In view of the toroidal and poloidal periodicity of the perturbation, non-uniformities in the plasma response could be expected. Such effects can be explored by changing the phasing of the DED fields and thus sweeping the Poincar structure with respect to the probe. A further issue concerns the radial mapping of points with and without DED. This is thought to be less of a problem in the SOL, where the blobs only arrive after integrating and smoothing out most of the fine structure of the magnetic perturbation.

\section{RESULTS AND DISCUSSION}

\subsection{Blob identification and characterization}

In order to gain insight into the basic features of the blob transport, we first look at the $I_{s}$ data of Fig. 1 in the ohmic phase before the DED. The raw signal is depicted in Fig. 3(b) for 

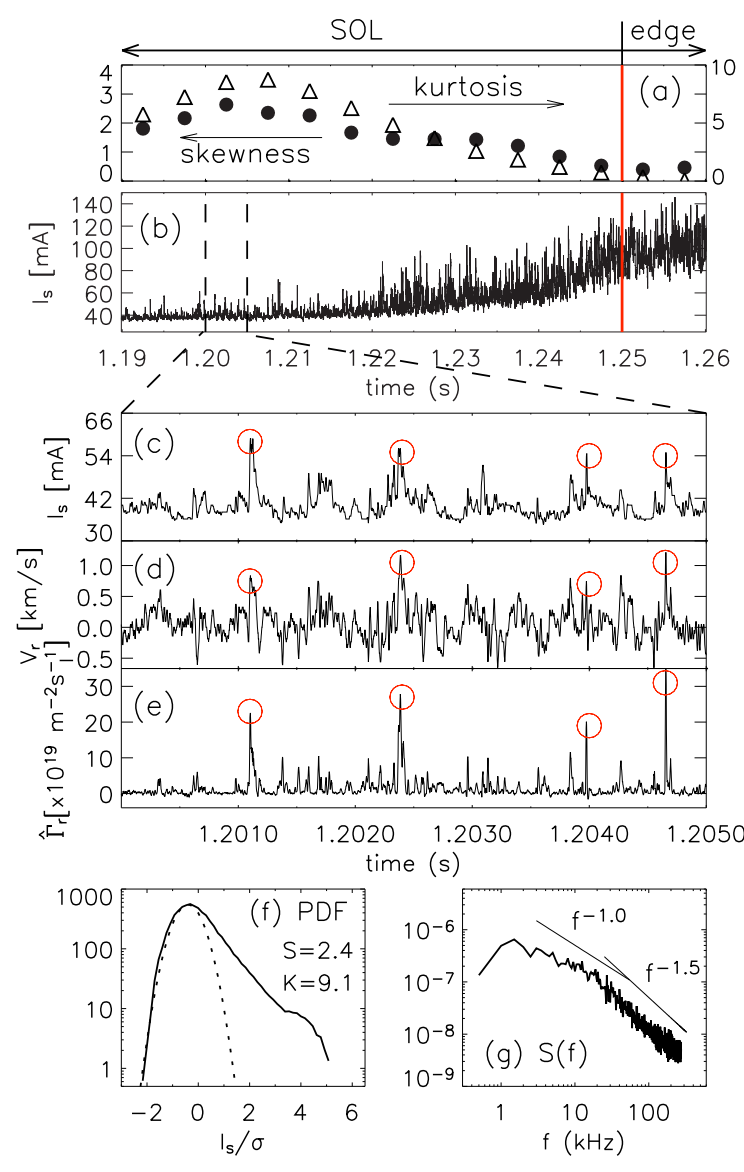

FIG. 3: Time history of $I_{s}$ signal (shot No. 101798) measured in the scrape-off layer (SOL) and plasma edge (b) and corresponding skewness (solid circles) and kurtosis (triangles) of the $I_{s}$ data (a). Shown in (c)-(e) are zooms of $I_{s}, V_{r}$, and $\tilde{\Gamma}_{r}$ signals in a $5 \mathrm{~ms}$ time window. (f) and (g) are the $\mathrm{PDF}$ of $I_{s}$ (normalized to the standard deviation) and frequency spectrum of $I_{s}$ measured in the SOL $(1.08 \geq \mathrm{r} / a \geq 1.05)$. The dotted curve in (f) is the best Gaussian-fit.

a radial range of $1.1 \geq \rho \geq 0.98$, including the SOL and the plasma edge. A lot of intermittent $I_{s}$ " bursts" are seen extending 4-5 cm deep into the SOL. Since $\tilde{I}_{s} / I_{s}=\tilde{n} / n+0.5 \tilde{T}_{e} / T_{e}$, the bursts in $I_{s}$ mainly reflects bursts (blobs) in density. The fluctuation data were digitized at a rate of $500 \mathrm{kHz}$.

The bursty behavior suggests a departure from a random diffusive process in the fluctuations. To be more quantitative in this interpretation, we evaluate the third (skewness) and fourth (kurtosis) order moments of the $I_{s}$ signal as plotted in Fig. 3(a). The skewness $(S)$ and kurtosis $(K)$ reflect respectively the degree of "asymmetry" and "peakedness" of a distribution of fluctuations [5, 7]. For a Gaussian signal, $S=K=0$, whereas for others 
the deviation from 0 indicates a higher degree of non-Gaussianity. Apparently, the increase of the $S$ and $K$ values from the edge to the SOL indicates that the intermittency prevails mainly in the SOL while in the edge the fluctuations are close to Gaussian statistics. To have a closer view on the intermittent bursty events and their impact on transport, we plot a zoom of $I_{s}$ for a $5 \mathrm{~ms}$ time window in Fig. 3(c), together with corresponding time traces of $V_{r}$ and $\tilde{\Gamma}_{r}$ signals in (d) and (e), respectively. $V_{r}$ is the radial speed of the blobs estimated by $V_{r}=\vec{E}_{\theta} \times \vec{B} / B^{2}$ ( $E_{\theta}$ is measured by two poloidally separated probe pins, as described in Section 2). The turbulent particle flux, $\tilde{\Gamma}_{r}$, is calculated by $\tilde{\Gamma}_{r}=\left\langle\tilde{n} \tilde{E}_{\theta}\right\rangle / B$, where the density fluctuations $\tilde{n}$ are computed from the $I_{s}$ fluctuations $\left(\tilde{I}_{s}\right)$ and the electron temperature

fluctuations $\tilde{T}_{e}$. $\langle\ldots\rangle$ denotes an ensemble average. The $\tilde{T}_{e}$ is obtained from a triple-probe configuration $[14,17]$.

In Figs. 3(c)-(e), we can see that at almost each density "spike" (marked by red circles), there exists a burst of radial speed, $V_{r} \sim 1 \mathrm{~km} / \mathrm{s}$, which eventually induces a large ejection of mass. It has been generally found that in the SOL the large burst fluctuations $(\geq 2.5$ standard deviation) account for nearly $40-50 \%$ of the total transport $[6,7]$. Because of the positive bursts, the PDF (probability distribution function) of $I_{s}$ measured in the far SOL exhibits a strong non-Gaussianity with high values of skewness and kurtosis, as seen in Fig. 3(f). The corresponding frequency spectrum of the $I_{s}$ fluctuations is shown in Fig. $3(\mathrm{~g})$. The power-law dependence in the spectrum reveals that the intermittency does not occur periodically, but is well embedded in a "sea of turbulence". The time-asymmetry in each $I_{s}$ burst, i. e., quick jump and slow decaying, suggests a radially moving structure with a steep front and a trailing wake. As such, this fluctuation-induced transport in the SOL is rightly termed "blob" transport. In the experiment, we also made a density scan and a safety factor scan and found that the degree of the SOL intermittency, as indicated by the $S$ and $K$ values, is enhanced with increasing plasma density and $q(a)$ [20].

\subsection{Reduction of blob transport during the application of the RMP}

The influence of the RMP on the SOL blob transport is illustrated in Fig. 4, where the radial profiles of $I_{s}, V_{r}$ and $\tilde{\Gamma}_{r}$ detected in the SOL and the plasma edge before (left column) and during (right column) the DED are compared. The probe data are taken from the first and the third plunge. As shown in Figs. 4(a) and (f), the amplitudes of blob bursts in $I_{s}$ 


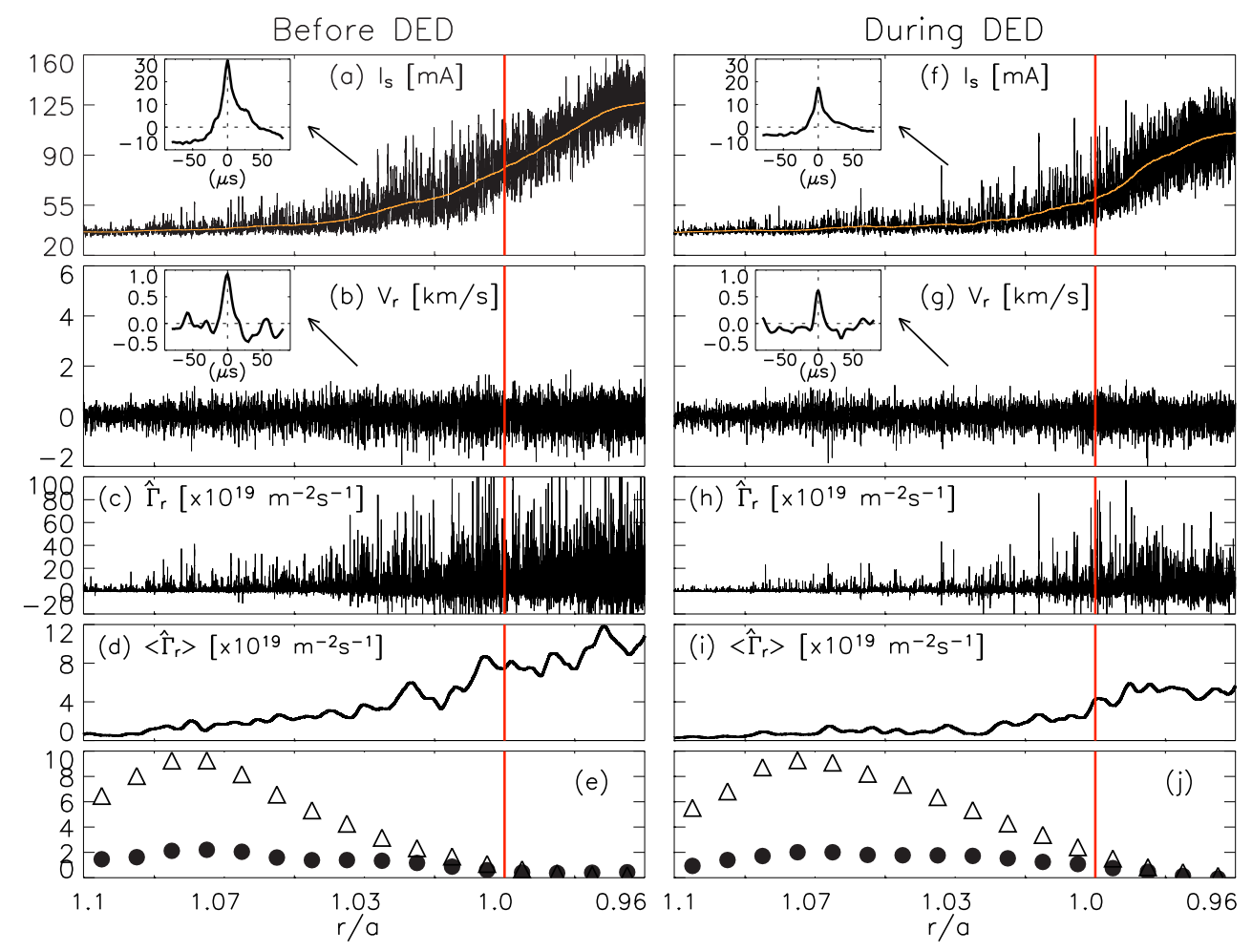

FIG. 4: Comparison of the $I_{s}, V_{r}$, and $\tilde{\Gamma}_{r}$ signals (versus normalized radial position, vertical red lines denote limiter location) measured in the SOL and edge before (left column) and during (right column) the DED (No. 101795). Plotted in (d) and (i) are the ensemble-average of turbulent flux. (e) and (j) show the skewness (solid circles) and kurtosis (triangles) of $I_{s}$. Corresponding parameters in the two columns are drawn in the same scale. The inset in (a) and (f) shows the auto-conditional-average of $I_{s}$ measured around $\mathrm{r} / a=1.03$ before and during the DED, respectively. The corresponding cross-conditional-average of $V_{r}$ is plotted in the inset of (b) and (g), respectively.

are decreased in the SOL in contrast to what happens in the edge. The corresponding $V_{r}$ is also reduced [see (b) and (g)]. The intermittency degree in the turbulent flux, $\tilde{\Gamma}_{r}$, is largely depressed [see (c) and (h)], at the same time as also the time-averaged turbulent flux is reduced, as shown in (d) and (i). The results thus clearly indicate a reduction by the RMP of the turbulent blob transport in the SOL, in agreement with theoretical simulations [21].

With an eye on elucidating the possible mechanisms, we first compared the radial profiles of the skewness and kurtosis of the $I_{s}$ signal before and during the DED. As seen in Figs. 4(e) and (j), with DED, there is almost no change of the $S$ and $K$ values in the SOL and the edge, which indicates that the bursty character of $I_{s}$ subsists with the RMP on. To gain more 

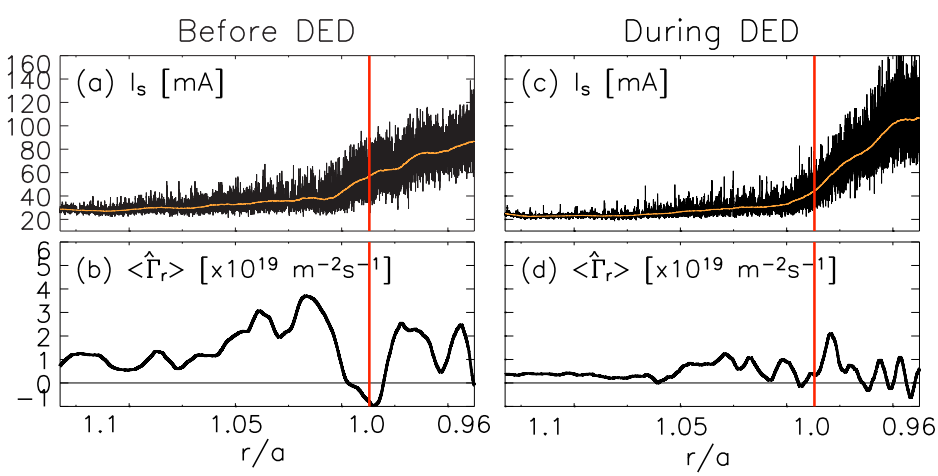

FIG. 5: Comparison of $I_{s}$ and turbulent flux signals in the SOL and the edge (vertical red lines denote limiter location) before (left column) and during (right column) the DED in a discharge where the edge density $\left(I_{s}\right)$ gradient increases during DED (No. 99630). The plasma parameters are similar to those of Fig. 4 but at a lower line-averaged density of $\bar{n}_{e}=1.5 \times 10^{19} \mathrm{~m}^{-3}$.

insight into exactly how the blob structure is affected by the RMP, we have made an autoconditional averaging of the intermittent bursts in $I_{s}$ and a cross-conditional averaging on the $V_{r}$ data by selecting the burst amplitudes larger than two times of the standard deviation in the $I_{s}$ signal. The technical details of the statistics have been described earlier $[2,6,7]$ and will not be repeated here. The auto-conditional average of $I_{s}$ and cross-conditional average of $V_{r}$ measured around $\mathrm{r} / a=1.03$ before and during the DED are shown in the insets of Figs. 4(a, f) and (b, g), respectively. From the conditional-averaged data, several features can be seen: (i) before and during DED, the $I_{s}$ burst shape is similar, confirming little change of $S$ and $K$ of the $I_{s}$ fluctuations, as already calculated and shown in Figs. 4(e) and (j); (ii) the $I_{s}$ burst amplitude is strongly reduced by the DED (from 30 to 17); (iii) the radial "passing time" of the blobs at the probe pin, defined as the width-at-half-height of the $I_{s}$ burst, is nearly halved from $60 \mu s$ to $30 \mu s$; (iv) $V_{r}$ is reduced during DED as exhibited by its conditional average data (Figs. 4(b) and (g)). Because of the decrease of both the "passing time" and of $V_{r}$, the blob size $\left(\approx\right.$ "passing time" $\left.\times V_{r}\right)$ is substantially reduced by the RMP. The observed decrease of the burst amplitude by RMP is therefore linked to a reduction of the blob size.

The observed turbulence changes induced by the RMP are quite robust and persist even under conditions where the edge equilibrium profiles react quite differently to the DED application, depending on discharge parameters and the wall-condition. In the case of Fig. 4, the edge density (or pressure) profile decreases around the limiter position (see yellow solid 

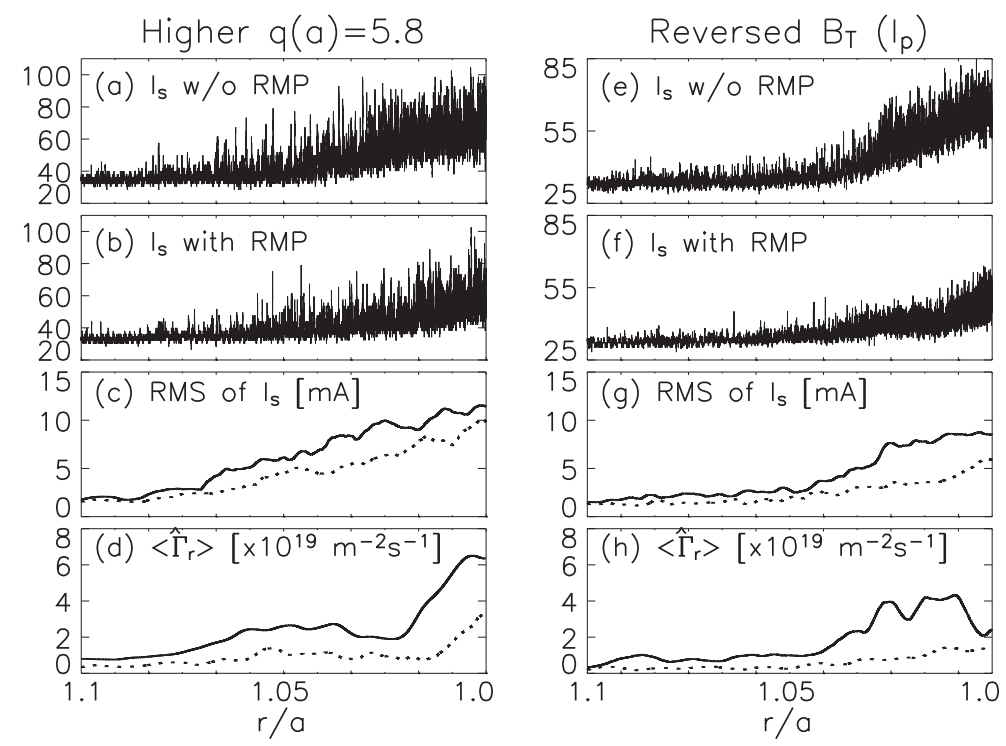

FIG. 6: Test of the RMP-phasing effect on the blob transport in the SOL in a higher $q(a)$ (left column, No. 101800: R/a@1.73/0.46 [m/m], $\left.B_{T}=1.9 \mathrm{~T}, I_{p}=200 \mathrm{kA}, \bar{n}_{e}=2.0 \times 10^{19} \mathrm{~m}^{-3}, I_{D E D}=6 \mathrm{kA}\right)$

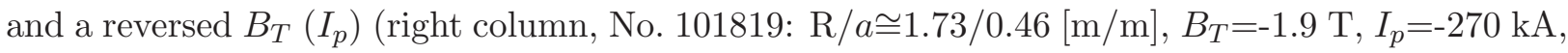
$\bar{n}_{e}=2.0 \times 10^{19} \mathrm{~m}^{-3}, I_{D E D}=6 \mathrm{kA}$ ) experiment. (a, b) and (e, f) show the raw data of $I_{s}$ signals $[\mathrm{mA}]$ without and with the RMP. Plotted in (c) and (g) are the relative RMS level of $I_{s}$ fluctuations without (solid curves) and with (dashed curves) the RMP. (d) and (h) are the time-averaged turbulent flux without (solid curves) and with (dashed curves) the RMP.

curves in Figs. 4(a) and (f)) when the RMP turns on. It might be argued that the decrease of the blob size in the SOL is simply caused by an inward shift of the density (or pressure) gradient. To rule that out, we compare in Fig. 5 with the data of another type of discharges where the $I_{s}$ gradient increases in the limiter region during the DED (see Figs. 5(a) and (c)). Most of the parameters of this discharge, belonging to a different campaign, are the same as those in Fig. 4, but for the plasma density which is $\bar{n}_{e}=3.0 \times 10^{19} \mathrm{~m}^{-3}$ for Fig. 4 and $1.5 \times 10^{19} \mathrm{~m}^{-3}$ for Fig. 5. The detailed reason for the difference in the global edge behaviour by the RMP is still under investigation at TEXTOR. Nevertheless, the turbulence results displayed are quite clear: as in the case of Fig. 4, also in Fig. 5, the blob amplitudes and the associated transport in the SOL are reduced.

In DIII-D, a toroidal non-uniform effect of the RMP on the edge fluctuations was observed [16]. It was therefore important to look for a possible similar effect in our experiment. As we only have a probe at one toroidal location, we changed the phasing of the DED fields 
with respect to this fixed position (thus substantially changing there the baseline topology of the Poincaré plot (see Fig. 2)). For this purpose, we have made several scans of the safety factor $(3.2 \leq \mathrm{q}(a) \leq 5.8)$ and of the DED current $(4-6 \mathrm{kA})$, we reversed $B_{T}\left(\right.$ and $I_{p}: I_{p}$ also reversed to maintain the pitch angle of the DED coil parallel to field lines around $q \cong 3$ surface in the plasma), and made these changes for several densities $\left((1.6-3.5) \times 10^{19} \mathrm{~m}^{-3}\right)$. From shot to shot, we changed one of the above quantities while in each case the global plasma parameters were all kept constant in time. However, in all cases we found the same tendency, independent of the phasing variation of the RMP: the blob size and the associated transport are reduced in the SOL. We can conclude that there is no toroidal non-uniform effect in TEXTOR. Illustrative examples can be found in Fig. 6 for a higher $q(a)=5.8\left(I_{p}\right.$ reduced to $200 \mathrm{kA}$ ) in the left column and a reversed $B_{T}\left(I_{p}\right)$ experiment in the right column, respectively. In both cases, as well from the raw data of $I_{s}$ plotted in Figs. 6(a, b) and (e, f) as from the relative RMS (root-mean-square) level of $I_{s}$ fluctuations drawn in (c) and (g), one can see a decrease of the blob amplitude in the SOL when the RMP turns on. At the same time, the time-averaged turbulent flux decreases with the RMP, as seen in Figs. 6(d) and (h). In a last further configuration test, we studied the transport in the $m / n=3 / 1$ DED mode of operation at $I_{D E D}<2 \mathrm{kA}$, to avoid triggering external tearing modes. Even at this low DED current, the blob amplitudes and turbulent transport in the SOL are also reduced by the RMP.

\subsection{Contributions to the understanding of blob transport and of blob transport} reduction caused by the RMP

In this section, we first compare our experimental results with predictions on blob formation and dynamics by theories based on the interchange instability [22-24]. The theory proposes that the blob structure develops on the base of a turbulent eddy inside (near) the separatrix, and is then driven radially outwards by the curvature drift on a dipolar vorticity. Earlier experiments on other devices have provided several supporting elements: (i) the intermittency is little recorded in the high-field side of the SOL, for example in the MAST tokamak [25], probably due to the good curvature; (ii) in DIII-D, the reversed $B_{T}$ experiment has testified the $\mathbf{B}$ curvature-polarization dynamics on blob structures [6]. In our experiment, as reported in section 3.1, blob transport in the low-field SOL is enhanced with 

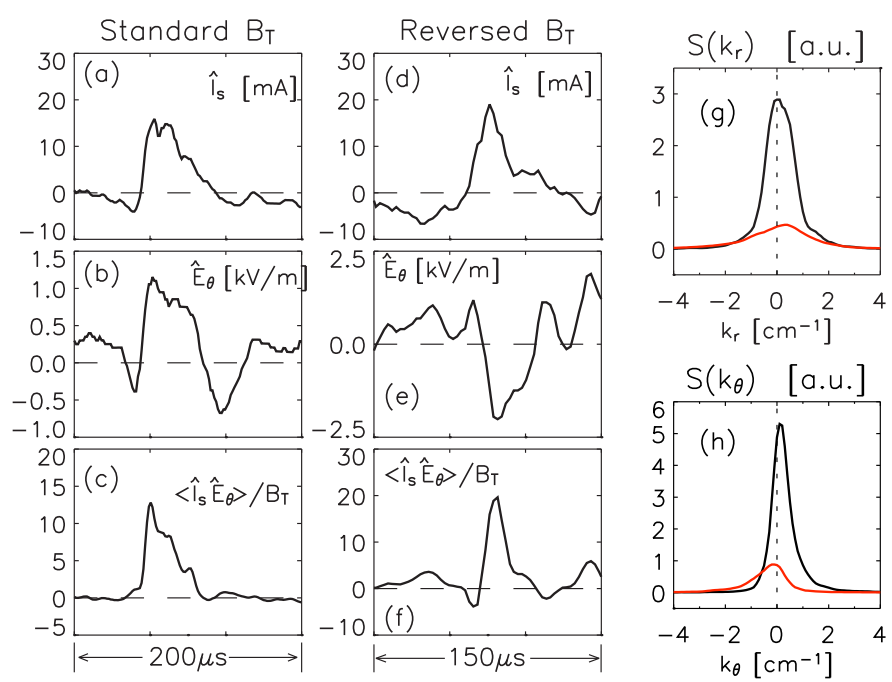

FIG. 7: (a-f) Comparison of the burst events of $\tilde{I}_{s}, \tilde{E}_{\theta}$, and $\left\langle\tilde{I}_{s} \tilde{E}_{\theta}\right\rangle / B_{T}$ signals measured in the SOL: (a)-(c) in standard $B_{T}$ and (d)-(f) in reversed $B_{T}$ ohmic discharge. Drawn in (g) and (h) are the radial and poloidal wave-number spectra measured in the ergodic region $(\mathrm{r} / a \approx 0.94)$ before (black curve) and during (red curve) the RMP.

increasing $q(a)$, possibly on account of a longer stay of blobs in the bad curvature zone. We also compared the blob dynamics in the standard and reversed $B_{T}$ experiments. Important fluctuation signals measured in the SOL in a standard $B_{T}$ and a reversed $B_{T}$ discharge are shown in Figs. 7(a)-(c) and (d)-(f), respectively. At one $I_{s}$ burst, a $B_{T}$ reversal results in an inversion of $E_{\theta}$ by $-\vec{\kappa} \times \vec{B}_{T}$ ( $\vec{\kappa}$ is curvature vector) while maintaining a radially-outward speed of $\vec{E}_{\theta} \times \vec{B}_{T} / B^{2}$ and hence turbulent flux of $\left\langle\tilde{I}_{s} \tilde{E}_{\theta}\right\rangle / B_{T}$. The results are consistent with those from DIII [6].

The impact of the RMP on turbulence structures could be multifold. In simulation [21], it is shown that before RMP the modes with $k_{/ /} \approx 0$ on rational surfaces are subject to strong instabilities whereas during RMP the modes are damped by resistivity because $k_{/ /}$ becomes finite and nonzero. With the RMP, a common feature observed in Tore Supra [26] and TEXTOR [14] is that in the ergodic zone the large-scale turbulence structures are strongly suppressed. In the present experiment, the radial and poloidal wave-number spectra, $S\left(k_{r}\right)$ and $S\left(k_{\theta}\right)$, in the ergodic region have also been calculated from potential fluctuations measured by two radially and poloidally separated probe pins using two-point cross-correlation techniques [27]. The results of $S\left(k_{r}\right)$ and $S\left(k_{\theta}\right)$ without (black curves) and with (red curves) the RMP are shown in Figs. 7(g) and (h), respectively. It is seen that 
with RMP, the fluctuation power for large-scale turbulence structures (small components of $k_{r}$ and $k_{\theta}$ ) is strongly suppressed in the ergodized area. Consequently, the blob size, based on large turbulence structures, is reduced by the RMP when the blobs develop and pass radially through the ergodic layer.

The influence of the RMP on the blob moving speed may be related to an increased sheath dissipation in the perturbed volume. According to the theory [22, 23], the plasma blobs decelerate owing to dissipation such as collision and sheath effects. With the RMP, the effective field line connection length at the plasma boundary inside the limiter is in general shortened, leading to an increase of the sheath dissipation and hence a reduction of the radial advection velocity [22]. This might explain the decrease of the blob moving speed provoked by the DED, as seen in Figs. 4(b) and (g).

\section{CONCLUSION}

In conclusion, the impact of the RMP on turbulent blob transport has been investigated in the SOL of TEXTOR during the DED experiment. In the ohmic phase without the RMP, the intermittent blobs exhibit high non-Gaussianity with high values of skewness and kurtosis in the density $\left(I_{s}\right)$ signal. The blob bursts extend 4-5 $\mathrm{cm}$ deep into the SOL with a radially-outward moving speed of $\sim 1 \mathrm{~km} / \mathrm{s}$ and thus constitute an important outflux. With the application of the RMP, the burst amplitudes, the number of large blobs and their radial drift velocity are all generally reduced, leading to a strong reduction of the blob transport in the SOL. In fact, such a reduction of transport should result in an increased particle confinement, commensurate with the drop in $D_{\alpha}$ emission during the DED phase, seen in figure 1. It should however be noted that the reduction of the turbulent transport is opposed by the extra parallel outflow that results from the DED application, a contribution which is difficult to ascertain quantitatively. The blob dynamics appears to be consistent with the paradigm of the radial motions of the blob structures driven by the interchange instability. The RMP suppresses large-scale turbulence eddies, and consequently, the blob size and burst amplitudes when the blobs pass radially through the stochastic region. The increased sheath dissipation by the RMP may meanwhile cause a reduction of their moving speed. The results provide new evidence for a controlling role of the RMP on the edge transport and could bear possible implications for a decrease of the plasma-wall interaction 
in ITER.

[1] S. J. Zweben, Phys. Fluids 28, 974 (1985).

[2] A. V. Filippas et al., Phys. Plasmas 2, 839 (1995).

[3] M. Umansky et al., Phys. Plasmas 5, 3373 (1998).

[4] B. LaBombard, Nucl. Fusion 40, 2041 (2000).

[5] G. Y. Antar et al., Phys. Rev. Lett. 87, 065001 (2001).

[6] J. A. Boedo et al., Phys. Plasmas 8, 4826 (2001).

[7] Y. Xu et al., Plasma Phys. Control. Fusion 47, 1841 (2005).

[8] ITER Physics Basis, Nucl. Fusion 39, 2175 (1999).

[9] T. E. Evans et al., Phys. Rev. Lett. 92, 235003 (2004).

[10] Y. Liang et al., Phys. Rev. Lett. 98, 265004 (2007).

[11] Ph. Ghendrich et al., Nucl. Fusion 42, 1221 (2002).

[12] K. H. Finken et al., Plasma Phys. Control. Fusion 46, B143 (2004).

[13] Y. Xu et al., Phys. Rev. Lett. 97, 165003 (2006).

[14] Y. Xu et al., Nucl. Fusion 47, 1696 (2007).

[15] R. A. Moyer et al., Phys. Plasmas 12, 056119 (2005).

[16] J. A. Boedo et al., Report GA-A25852 (2007), submitted to the 34th EPS conference.

[17] H. Lin et al., Rev. Sci. Instrum. 63, 4611 (1992).

[18] M. W. Jakubowski et al., Phys. Rev. Lett. 96, 035004 (2006).

[19] S. S. Abdullaev et al., Phys. Plasmas 6, 153 (1999).

[20] Y. Xu et al., in the Proceedings of the 34th EPS conference on Plasma Physics (Warsaw, 2007), p1. 097.

[21] D. Reiser, Phys. Plasmas 14, 082314 (2007).

[22] O. E. Garcia et al., Phys. Plasmas 13, 082309 (2006).

[23] D. A. Russell et al., Phys. Rev. Lett. 93, 265001 (2004).

[24] A. V. Nedospasov et al., Nucl. Fusion 25, 21 (1985).

[25] G. Y. Antar et al., Phys. Plasmas 12, 032506 (2005).

[26] J. Payan et al., Nucl. Fusion 35, 1357 (1995).

[27] J. M. Beall, Y. C. Kim, and E. J. Powers, J. Appl. Phys. 53, 3933 (1982). 